\title{
Front Matter: Vol. 12083
}

, "Front Matter: Vol. 12083," Proc. SPIE 12083, Thirteenth International Conference on Graphics and Image Processing (ICGIP 2021), 1208301 (16 February 2022); doi: 10.1117/12.2628197

SPIE Event: Thirteenth International Conference on Graphics and Image SPIE. Processing (ICGIP 2021), 2021, Kunming, China 


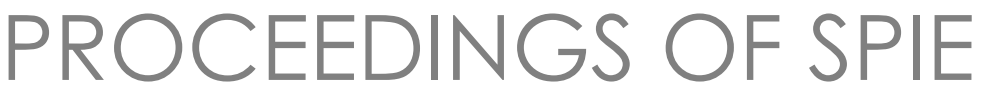

\title{
Thirteenth International Conference on Graphics and Image Processing (ICGIP 2021)
}

\author{
Liang Xiao \\ Dan Xu \\ Editors
}

18-20 August 2021

Kunming, China

Hosted by

Yunnan University (China)

Organized by

School of Information Science \& Engineering, Yunnan University (China)

Co-sponsored by

Foshan University (China)

Chang'an University (China)

Xi'an University of Technology (China)

Northwest A\&F University (China)

Sichuan University (China)

Ocean University of China (China)

University of Portsmouth (China)

China Society of Image and Graphics (China)

China Graphics Society (China)

Published by

SPIE

Volume 12083 
The papers in this volume were part of the technical conference cited on the cover and title page. Papers were selected and subject to review by the editors and conference program committee. Some conference presentations may not be available for publication. Additional papers and presentation recordings may be available online in the SPIE Digital Library at SPIEDigitalLibrary.org.

The papers reflect the work and thoughts of the authors and are published herein as submitted. The publisher is not responsible for the validity of the information or for any outcomes resulting from reliance thereon.

Please use the following format to cite material from these proceedings:

Author(s), "Title of Paper," in Thirteenth International Conference on Graphics and Image Processing (ICGIP 2021), edited by Liang Xiao, Dan XU, Proc. of SPIE 12083, Seven-digit Article CID Number (DD/MM/YYYY); (DOI URL).

ISSN: 0277-786X

ISSN: 1996-756X (electronic)

ISBN: 9781510650428

ISBN: 9781510650435 (electronic)

Published by

SPIE

P.O. Box 10, Bellingham, Washington 98227-0010 USA

Telephone +1 3606763290 (Pacific Time)

SPIE.org

Copyright @ 2022 Society of Photo-Optical Instrumentation Engineers (SPIE).

Copying of material in this book for internal or personal use, or for the internal or personal use of specific clients, beyond the fair use provisions granted by the U.S. Copyright Law is authorized by SPIE subject to payment of fees. To obtain permission to use and share articles in this volume, visit Copyright Clearance Center at copyright.com. Other copying for republication, resale, advertising or promotion, or any form of systematic or multiple reproduction of any material in this book is prohibited except with permission in writing from the publisher.

Printed in the United States of America by Curran Associates, Inc., under license from SPIE.

Publication of record for individual papers is online in the SPIE Digital Library.

\section{SPIE. DIGITAL}

Paper Numbering: A unique citation identifier (CID) number is assigned to each article in the Proceedings of SPIE at the time of publication. Utilization of CIDs allows articles to be fully citable as soon as they are published online, and connects the same identifier to all online and print versions of the publication. SPIE uses a seven-digit CID article numbering system structured as follows:

- The first five digits correspond to the SPIE volume number.

- The last two digits indicate publication order within the volume using a Base 36 numbering system employing both numerals and letters. These two-number sets start with 00, 01, 02, 03, 04, 05, 06, 07, 08, 09, 0A, OB ... 0Z, followed by 10-1Z, 20-2Z, etc. The CID Number appears on each page of the manuscript. 


\section{Contents}

\section{Part One}

\section{COMPUTER AND IMAGE PROCESSING}

1208302 Development of fault tree analysis and calculation software based on VC [12083-23]

1208303 Optimized firefly algorithm based on levy flight and Gaussian inertial weight [12083-40]

$1208304 \quad$ Robust adaptive graph learning with manifold constraints for subspace clustering [12083-51]

1208305 Application of XGBoost and traditional interpolation methods on wind fields in offshore areas of China [12083-54]

1208306 Stacked multi-modal refining and fusion network for visual entailment [12083-57]

1208307 Aggregating region context information for semantic segmentation [12083-100]

1208308 Design of depression assessment and early warning system for college students based on machine learning and daily health data [12083-131]

$1208309 \quad$ Learning class predication distributions with noisy labels [12083-152]

\section{COMPUTER PHOTOGRAPHY AND OPTICAL IMAGING TECHNOLOGY}

12083 OA The real-time measurement of the length of long line based on epipolar constraint [12083-46]

12083 OB Robust speckle-autocorrelation non-line-of-sight imaging with generative adversarial networks [12083-99]

12083 OC Learning structure-aware transformations for arbitrary image style transfer [12083-108]

12083 OD Binary optical time measurement based on image processing [12083-124]

12083 OE Hyperspectral pansharpening via deep detail injection network [12083-139]

12083 OF Fast external calibration of camera based on point set matching [12083-53]

12083 OG Hyperspectral image super-resolution via deep image gradient guided residual dense network [12083-148] 
$12083 \mathrm{OH} \quad$ Joint learning of latent representation and global similarity for multi-view image clustering [12083-20]

12083 Ol Human pose estimation based on manifold Gaussian process with depth images [12083-14]

12083 0J Detail preserving depth prediction from a single image based on multi-scale deep network and gradient network [12083-34]

12083 OK Adaptive pixelwise inference multi-view stereo [12083-77]

$12083 \mathrm{OL} \quad$ Visual knowledge mapping analysis of research of digital image processing [12083-122]

DIGITAL IMAGE PROCESSING AND APPLICATION

12083 OM Unsupervised generative adversarial network for style transfer using multiple discriminators [12083-36]

12083 ON Siamese dual path aggregation network for object tracking [12083-86]

1208300 A feature attention dehazing network based on U-net and dense connection [12083-87]

12083 OP Matrix completion via local density loss optimization [12083-112]

12083 OQ Implementation of star centroid extraction based on FPGA [12083-130]

12083 OR A real-time detection network for surface defects of mobile phone lens [12083-138]

\section{FEATURE ENHANCEMENT AND FEATURE FUSION}

12083 OS Dual-station stepped-frequency continuous waves MIMO radar for through-wall life detection using image fusion [12083-22]

12083 OT Multi-scale features enhanced sentiment region discovery for visual sentiment analysis [12083-47]

12083 OU Long-term correlation filter tracking algorithm based on adaptive feature fusion [12083-52]

12083 OV A lightweight road defect detection method based on multi-scale hybrid feature fusion [12083-104] 
12083 OW Siamese network tracker with channel attention mechanism [12083-143]

12083 OX Convolutional neural network based on feature enhancement and attention mechanism for Alzheimer's disease prediction using MRI images [12083-145]

12083 OY Multi-channel image fusion in ultraviolet dynamic detection on electric railways [12083-74]

\section{IMAGE ANALYSIS AND TRANSFORMATION}

$1208302 \quad$ An effective color correction method for underwater image [12083-6]

$1208310 \quad$ Texture image retrieval based on statistical modeling of all subbands in the complex transform domain [12083-30]

1208311 Sand-dust image enhancement based on color correction and haze removal [12083-33]

$1208312 \quad$ Multi-channel image inpainting algorithm based on edge prediction [12083-41]

$1208313 \quad$ Encoder-decoder network with self-attention module for image restoration [12083-76]

$1208314 \quad$ Blind restoration of vertical motion blurred image based on point spread function estimation [12083-102]

$1208315 \quad$ Uneven illumination image matching algorithm combined with single-parameter homomorphic filtering [12083-115]

1208316 A multi-scale threshold spiking mechanism based on spike camera [12083-120]

1208317 An adaptive median filtering denoising algorithm for pepper and salt noised image [12083-123]

\section{IMAGE CLASSIFICATION}

1208318 Subspace representation based on pairwise linear regression for large scale image set classification [12083-19]

$1208319 \quad$ Potato skin defect detection and classification through image processing [12083-21]

12083 lA Efficient 3D residual network on MRI data for neurodegenerative disease classification [12083-42]

12083 1B Fine-grained object classification based on block diagonal feature and ensemble learning [12083-45]

12083 IC HA-GCN: an ALS point cloud classification method based on height-aware graph convolution network [12083-75] 
12083 ID Spatially bound categorical attention network for semantic segmentation [12083-89]

\section{Part Two}

\section{IMAGE RECONSTRUCTION AND POINT CLOUD DATA PROCESSING}

12083 1E Road extraction from 3D point clouds based on the difference of normal vector [12083-24]

12083 IF Scattered point cloud simplification algorithm based on FPFH and FPS [12083-13]

12083 IG 3D mesh reconstruction of indoor scenes from a single image in-the-wild [12083-93]

$120831 \mathrm{H} \quad$ Deep learning radiomics model based on contrast-enhanced T1-weighted image multi-plane reconstruction for prediction of glioma grading [12083-97]

1208311 3D reconstruction of cross-scale narrow space with thin structure [12083-101]

$120831 \mathrm{~J} \quad$ A single image super-resolution reconstruction based on fusion [12083-150]

12083 1K 1-point sample consensus on correspondence set for 3D point cloud registration [12083-32]

12083 IL Divide-and-conquer for holistic and expressive 3D human body reconstruction from a single RGB image [12083-94]

\section{IMAGE SEGMENTATION AND CLASSIFICATION}

12083 IM Multiple GANs guided by self-attention mechanism for automatic cardiac image segmentation [12083-15]

12083 iN Research on parallel technology of sea and land segmentation based on deep learning [12083-106]

1208310 DFM-Net: a contextual inference network for T2-weighted image segmentation of the pelvis [12083-121]

12083 IP Image segmentation application combining moving grid method and level set method [12083-125]

$120831 Q \quad$ 3D U-Net with trans-coder for brain tumor segmentation [12083-133]

\section{IMAGE TRANSMISSION AND IMAGE SECURITY}

12083 IR SA-UNet for face anti-spoofing with depth estimation [12083-9] 
12083 is Efficient multi-step reasoning attention network for visual question answering [12083-38]

12083 1T An effective image encryption algorithm based on a novel chaotic map [12083-63]

$1208314 \quad$ A graph convolutional neural network model for trajectory prediction [12083-70]

12083 IV Chaotic image encryption algorithm based on R-order Chebyshev composite map [12083-83]

12083 IW Layout-aware bidirectional transfer network for fashion landmark detection [12083-105]

12083 1X A remote sensing image processing method based on color restoration and enhancement [12083-128]

\section{INTELLIGENT RECOGNITION TECHNOLOGY AND APPLICATION}

$120831 Y \quad$ Using multi-feature fusion and XGBoost to recognize the pedestrian with multiple angles in different videos [12083-17]

$1208312 \quad$ An overview of traffic sign detection and recognition algorithms [12083-37]

1208320 A multi-scale deformable convolution network model for text recognition [12083-56]

$1208321 \quad$ Learning multi-level representations for image emotion recognition in the deep convolutional network [12083-91]

1208322 A no reference image quality assessment method based on RepVGG [12083-110]

$1208323 \quad$ Replacing speaker-independent recognition task with speaker-dependent task for lip-reading using First Order Motion Model [12083-154]

$1208324 \quad$ Video-based multimodal personality analysis [12083-156]

MEDICAL IMAGING AND VIRTUAL TECHNOLOGY

1208325 Measurement of endometrial thickness using deep neural network with multi-task learning [12083-3]

$1208326 \quad$ Noise reduction in dental CT images based on generative adversarial network [12083-4]

1208327 A virtual experimental platform based on MR glasses guided by Kano's Theory [12083-12]

1208328 Adaptive weighted PET/SPECT and MR medical image fusion based on nonsubsampled shearlet transform [12083-111] 
1208329 The application of virtual reality technology in the middle school research travel course: taking Suzhou Shantang Street as an example [12083-79]

$120832 \mathrm{~A}$ An automatic matching interactive and visualization system for virtual martial arts [12083-82]

12083 2B PANN: an efficient parallel neural network based on the attentional mechanism for predicting Alzheimer's disease [12083-114]

$120832 \mathrm{C}$ Distributed management of virtual reality digital assets for online experimental education [12083-147]

\section{PATTERN RECOGNITION}

12083 2D A gesture recognition smart media interactive application [12083-16]

$120832 \mathrm{E} \quad$ Review of human action recognition based on improved deep learning methods [12083-18]

$120832 F \quad$ Image recognition of sandstone slice based on a lightweight network [12083-43]

120832 G Mini-TKAGCN: a lightweight graph convolutional network via temporal kernel attention for skeleton-based action recognition [12083-132]

$120832 \mathrm{H} \quad 3 \mathrm{DCNN}-$ based mouth shape recognition for patient with intractable neurological diseases [12083-155]

$1208321 \quad$ A review of action recognition methods based on skeleton data [12083-31]

$120832 \mathrm{~J} \quad$ Summary of fine-grained image recognition based on attention mechanism [12083-68]

\section{TARGET DETECTION AND METHOD}

12083 2K A weakly supervised object detection method based on attention mechanism [12083-7]

$120832 \mathrm{~L} \quad$ Research on detection methods of high beam in night driving vehicles [12083-35]

$120832 \mathrm{M}$ An aggregation context network for detecting small ships in remote sensing images [12083-61]

$120832 \mathrm{~N}$ Small infrared maritime target detection based on gradient amplitude difference and multidimensional dissimilarity measure [12083-64]

1208320 Small-scale pedestrian detection based on multi-level feature fusion [12083-1 19]

$120832 \mathrm{P} \quad$ Image edge detection based on Krisch operator and gray correlation analysis [12083-149] 
VIDEO TECHNOLOGY AND IMAGE QUALITY EVALUATION

$120832 \mathrm{~A} \quad$ A semantic simultaneous localization and mapping system based on DeeplabV3+ [12083-10]

$120832 R \quad$ A fourth derivative based tool for pulse signal special point identification [12083-25]

$1208325 \quad$ Application of spatiotemporal convolution network for precipitation nowcasting [12083-65]

$120832 \mathrm{~T} \quad$ Research on fast motion estimation in H264 coding [12083-96]

$120832 \mathrm{U} \quad$ An assessment and grading method of ultra-HD TVs based on subjective and objective evaluation [12083-98]

$120832 \mathrm{~V} \quad$ Assessment of image sharpness evaluation methods and image sharpness changes in GF-4 satellite time-series data [12083-127] 
Proc. of SPIE Vol. 12083 1208301-10 Downloaded From: https://www.spiedigitallibrary.org/conference-proceedings-of-spie on 26 Apr 2023
Terms of Use: https://www.spiedigitallibrary.org/terms-of-use 